\title{
Overexpression of Malic Enzyme 2 Indicates Pathological and Clinical Significance in Oral Squamous Cell Carcinoma
}

\author{
Jun-Jie Zhou ${ }^{1}$, Yao Xiao ${ }^{1}$, Hao Li ${ }^{1}$, Cong-Cong Wu ${ }^{1}$, De-Run Chen ${ }^{1}$, Lei Chen ${ }^{1}$, Wei-Wei Deng', Wen-Feng \\ Zhang ${ }^{2}$, and Zhi-Jun Sun $1,2^{\boxplus}$ \\ 1. The State Key Laboratory Breeding Base of Basic Science of Stomatology (Hubei-MOST) \& Key Laboratory of Oral Biomedicine Ministry of Education, \\ School \& Hospital of Stomatology, Wuhan University, Wuhan, China \\ 2. Department of Oral Maxillofacial-Head Neck Oncology, School and Hospital of Stomatology, Wuhan University, 237 Luoyu Road, Wuhan 430079, Hubei \\ Province, China \\ $\bowtie$ Corresponding authors: Prof. Zhi-Jun Sun and Wen-Feng Zhang. Emails: sunzj@whu.edu.cn; zhangwf59@whu.edu.cn; Fax: +862787873260
}

( ) The author(s). This is an open access article distributed under the terms of the Creative Commons Attribution License (https://creativecommons.org/licenses/by/4.0/). See http://ivyspring.com/terms for full terms and conditions.

Received: 2020.01.10; Accepted: 2020.02.25; Published: 2020.03.05

\begin{abstract}
Our study investigated the expression of malic enzyme 2 (ME2) in human oral squamous cell carcinoma (OSCC) and associated pathological and clinical pattern. We demonstrated that human OSCC tissues expressed a high level of ME2, and the overexpression of ME2 is closely connected to a high pathological grade, lymphatic metastasis, large tumor size and human papillomavirus (HPV) $(P<0.001)$. Similarly, high levels of ME2 expression in OSCC tissue were shown to be correlated with poor prognosis $(P<0.05)$. The expression of ME2 was correlated with Slug, SOX2, and aldehyde dehydrogenase-1 (ALDH1) immunoreactivity.ME2 was shown to be overexpressed in OSCC tissue and indicated a poor prognosis for OSCC. ME2 may be correlated with several immune markers.
\end{abstract}

Key words: malic enzyme 2, oral squamous cell carcinoma, prognosis, tissue microarrays

\section{Introduction}

OSCC is one of the most common human malignancies worldwide [1-3]. In America, the incidence rate of oropharyngeal squamous cell carcinoma has been increasing sharply [4]. It is a cancer that originates in moist, mucosal surfaces lined by squamous cells [5]. It has been shown that this incidence occurs with smoking, alcoholic beverage consumption, and human papillomavirus (HPV) infection [2, 4]. Despite therapeutic advances, the overall 5-year survival rate for oral cancer has improved marginally and has remained unchanged over the past few decades [2, 4, 6]. Consequently, there is a high unmet medical need for efficacious therapy against OSCC.

Recently, targeting cancer metabolism has become a propitious strategy for the development of selective antineoplastic agents [7-9]. Great progress has been made in this field. In contrast, targeting cancer metabolism for treating OSCC has not been thoroughly reported, and multiple metabolites and metabolic enzymes of OSCC remain unelucidated.

There are three isoforms in the family of malic enzymes, comprising a cytosolic isoform (ME1) and two redundant mitochondrial isoforms (ME2 and ME3) [10-12]. ME2 has higher efficiency and activity than ME3. In addition, ME2 is the main mitochondrial isoform in various cells $[13,14]$. Previous work has indicated that the expression level of ME2 is noticeably increased in malignant human tissues, such as pancreatic cancer, lung cancer, head and neck squamous cell carcinoma (HNSCC), etc [15-17]. According to the TCGA database, previously published studies on HNSCC have shown that overexpression of ME2 predicts poor survival [15]. Woo $\mathrm{SH}$ et al. showed that metformin-induced senescence is enhanced using depletion and that metformin inhibits ME2 expression [15]. Until now, 
no field experiments of ME2 in OSCC tumors have been reported.

The primary goal of this research was to characterize a significant increase in the ME2 expression level in human OSCC tissue and to interpret its correlation with clinicopathological significance and prognosis. In addition, the correlation between ME2 and Slug, SOX2, and ALDH1 was evaluated.

\section{Materials and Methods}

\section{Human OSCC tissue microarrays}

Custom-made human OSCC tissue microarrays were obtained from patients who take surgery at the Department of Oral and Maxillofacial Surgery, School and Hospital of Stomatology Wuhan University. All surgical procedures were approved by School and Hospital of Stomatology of Wuhan University Medical Ethics Committee. We used the guidelines of the Union for International Cancer Control (UICC 2016) to determine the TNM stages of human OSCC. HPV was diagnosed by the HPV DNA in situ hybridization technique and p16 immunostaining.

Custom-built tissue microarray slides (T12-412TMA2, T15-411, and T17-790, as previously described [18]) included 176 primary OSCC tissues (cases with preoperative radiation and/or chemotherapy were excluded), 42 normal oral mucosal tissues, 69 oral epithelial dysplasia tissues, 59 metastatic lymph tissues, 20 tissues from OSCC patients who obtained preoperative TPF-inductive chemotherapy (cisplatin, docetaxel, and fluorouracil) [19], 15 tissues from OSCC patients who obtained preoperative radiotherapy treatment, and 24 tissues from patients with recurring OSCC. There were 12 patients with an HPV infection, 98 patients who smoke, and 82 patients who drink alcohol included in this study.

\section{Immunohistochemical staining}

The methods and processes of immunohistochemical staining were performed as previously described [20]. Briefly, all OSCC tissue microarrays were cut into $4-\mu \mathrm{m}$ sections. Slides were deparaffinized by dimethylbenzene and hydrated by ethyl alcohol sequentially. Antigen retrieval was conducted using sodium citrate $(\mathrm{pH}=6.0)$ under high pressure. Then, the sections were incubated to block endogenous peroxidase activity using hydrogen superoxide. After blocking with goat serum at $37^{\circ} \mathrm{C}$ for $20 \mathrm{~min}$, the slides were incubated with the following antibodies: ME2 (Cell Signaling Technology, USA), Slug (Cell Signaling Technology, USA), SOX2 (Cell Signaling Technology, USA), and ALDH1(Cell Signaling Technology, USA) at $4^{\circ} \mathrm{C}$ overnight. After washing the slides with PBS, an appropriate secondary biotinylated immunoglobulin $\mathrm{G}$ antibody solution and an avidin/biotin/peroxidase reagent were added to the slides. Immunohistochemical staining was accomplished with diaminobenzidine and then gently counterstained with hematoxylin (Invitrogen, Waltham, MA, USA).

\section{Immunohistochemistry scoring and hierarchical clustering}

Scoring of the immunohistochemistry results, hierarchical clustering, and data visualization were accomplished as previously described [21]. The immunohistochemical stained samples were scanned by an Aperio ScanScope CS whole slice scanner (CA, USA) and Aperio quantification software (version9.1) with background subtraction as previously described, and the slides were analyzed by pixel quantification. The nuclear and membrane staining were quantified using the following formula: $(3+)^{*} 3+(2+)^{*} \times 2+(1+)^{*}$ 1 , as described in a previous study[22]. Total intensity/total cell number was calculated as a histoscore from pixel quantification. Microsoft Excel was used to convert the scaled values of expression scores. Cluster 3.0 was used for hierarchical analysis with the average linkage based on Pearson's correlation coefficient for hierarchical analysis, and Java TreeView1.0.5 [23] was used for direct visualization [24] of the results.

\section{Western blot analysis}

The tumor and Oral Mucosa tissue were carefully obtained from patients after surgical resection at the Department of Oral and Maxillofacial Surgery, School and Hospital of Stomatology Wuhan University and Western blot was performed as previously described[25]. The primary antibodies used were as follows: ME2. GAPDH was used as a control.

\section{Statistical analysis}

Statistical comparisons were made using GraphPad Prism 8.01 for Windows (GraphPad Software, Inc., La Jolla, CA). One-way ANOVA followed by Tukey's post-hoc multiple comparison test and unpaired t-test were used to analyze the significance in immunohistochemical staining. Kaplan- Meier analysis was used for plot survival curves, and the significance of differences was assessed using the log-rank test. The cutoff was confirmed from the website Cutoff Finder according to a previously reported protocol [26]. These variables were translated into a clinical decision, and the cutoff point was used to stratify patients into two groups. The Cox multivariate proportional hazards regression model was used to assess the significance of survival analysis. All data are presented as the means \pm SEM, and statistical significance was considered as $\mathrm{P}<0.05$. 


\section{Results}

\section{ME2 overexpression in human OSCC}

To investigate ME2 expression level in human OSCC, we used Aperio ScanScope to quantify the immunohistochemical staining results of the whole slides, which characterized the expression of ME2 in the tissue microarray (Fig. 1A, 1B). Indeed, a high expression level of ME2 was observed in human primary OSCC tissue $(n=176)$ compared with normal oral mucosal tissue $(n=42, P<0.05$, Fig. $1 C)$, whereas the difference in the expression level of ME2 between the OSCC and dysplasia tissues was not significant (n $=69$, P > 0.05, Fig. 1C).

Additionally, we explored the prognosis value of ME2 in OSCC by Kaplan-Meier method and log-rank test analysis, and the cutoff of ME2 expression (histoscore $=23.91$ ) was decided by Cutoff Finder [26] . Statistical analysis demonstrated that ME2 expression was associated with poor prognostic implications in patients with OSCC ( $\mathrm{P}<0.05$, Hazard ratio: 2.114 , 95\% CI: 1.066 to 4.192, Fig 1D). Meanwhile, the result of multivariate Cox proportional hazard model analysis confirmed the validity of ME2 was prognostically significant for patient with OSCC $(\mathrm{P}<0.05$, Table 1$)$.

Table 1. Multivariate analysis for overall survival in primary OSCC patients (with ME2).

\begin{tabular}{lll}
\hline Parameters & HR $(\mathbf{9 5} \% \mathrm{CI})$ & $\boldsymbol{P}$ value \\
\hline Gender & $0.461(0.167-1.274)$ & 0.135 \\
Age & $0.701(0.334-1.469)$ & 0.347 \\
Smoking & $0.960(0.386-2.389)$ & 0.930 \\
Drinking & $1.388(0.597-3.225)$ & 0.446 \\
Pathological grade & & \\
II vs. I & $1.579(0.671-3.716)$ & 0.296 \\
III vs. I & $1.830(0.608-5.512)$ & 0.282 \\
Tumor size & & \\
T2 vs. T1 & $1.681(0.537-5.257)$ & 0.372 \\
T3 vs. T1 & $3.147(0.882-11.230)$ & 0.077 \\
T4 vs. T1 & $1.494(0.263-8.485)$ & 0.650 \\
Node stage & & \\
N1 vs. N0 & $0.570(0.204-1.592)$ & 0.283 \\
N2 vs. N0 & $2.210(0.739-6.607)$ & 0.156 \\
ME2 expression & $0.405(0.185-0.885)$ & $0.023^{*}$ \\
\hline
\end{tabular}

Cox proportional hazards regression model. HR hazard ration, 95\% CI 95\% confidence interval. ${ }^{*} P<0.05$
A

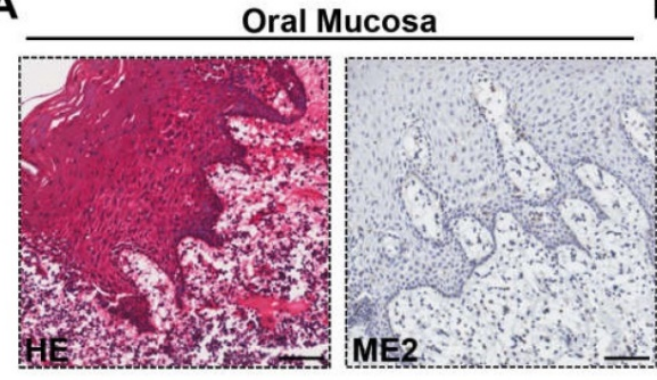

B

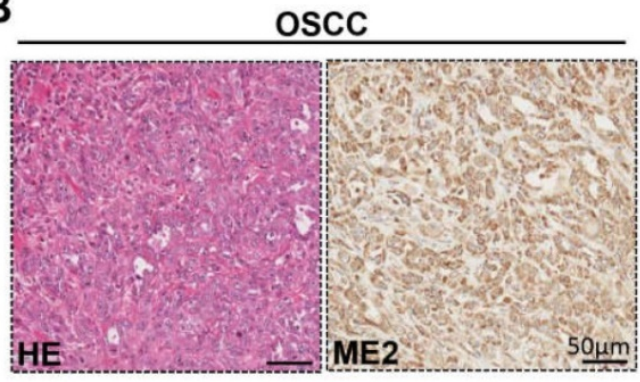

C

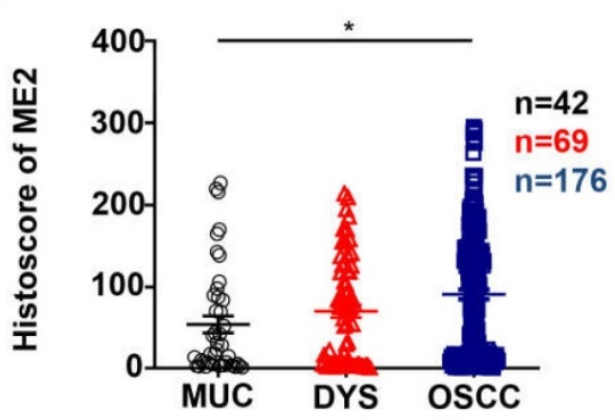

D

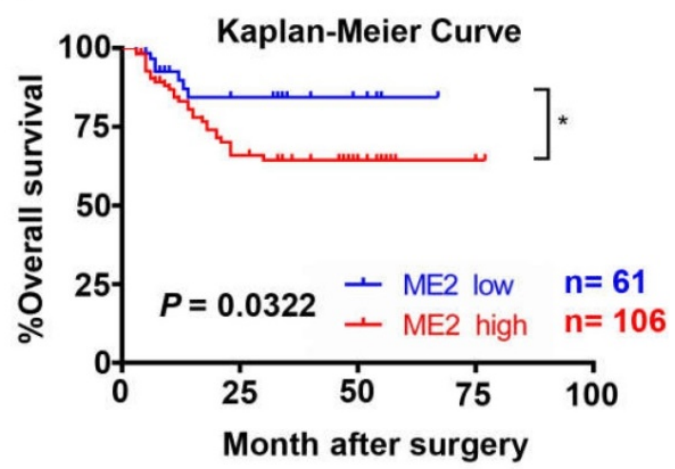

$\mathbf{E}$

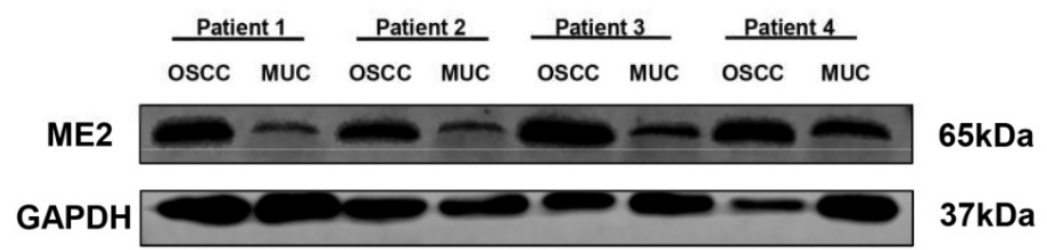

Figure 1. ME2 is expressed at a high level in oral squamous cell carcinoma. Representative hematoxylin-eosin (HE, left) and IHC (right) staining of ME2 in oral mucosal tissue(A) and in primary OSCC tissue(B). Scale bar: $50 \mu \mathrm{m}$. (C) Quantification of immunohistochemical histoscore of ME2 for oral mucosal tissue $(n=42)$, dysplasia tissue (DYS, $n=69)$ and primary oral squamous cell carcinoma tissue (OSCC, $n=176)$. (D) A Kaplan-Meier curve showing that OSCC patients with a low expression level of ME2 ( $=61)$ survive longer than patient with a high expression level of ME2 $(n=106)$, and log-rank analysis showed that the difference was significant $(P<0.05)$. $(E)$ Western blot analysis the protein expression of ME2 in primary OSCC tissue and oral mucosal tissue. GAPHD was used as loading control. 


\section{The expression of ME2 in OSCC was associated with pathological features}

To further confirm the relationship between the expression of ME2 and pathological features, we observed the ME2 expression was notably correlated with different pathological grade. ( $\mathrm{P}<0.05$, Fig. $2 \mathrm{~A})$. Notably, a high expression level was observed with different tumor sizes. $(\mathrm{P}<0.05$, Fig. $2 \mathrm{E})$. The result showed that a notable upregulation of ME2 expression was observed in primary tumor patients with pathologically positive lymph node status $(\mathrm{P}<$ 0.05 , Fig. 2D). Consequently, we comparatively assessed ME2 expression in human primary OSCC and lymph nodes. Interestingly, we discovered a borderline significant correlation between OSCC and $\mathrm{LN}(\mathrm{P}=0.1365$, Fig. $2 \mathrm{G})$. Another interesting finding was a comparable increase in the ME2 expression level in OSCC patients with HPV ( $\mathrm{P}<0.001$, Fig. 3B) but ME2 expression independent of smoking ( $\mathrm{P}>$ 0.05, Fig. 3C) or alcohol consuming ( $\mathrm{P}>0.05$, Fig. 3D).

\section{A}

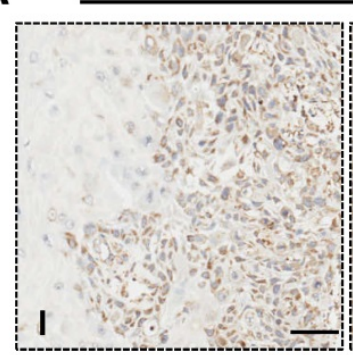

C
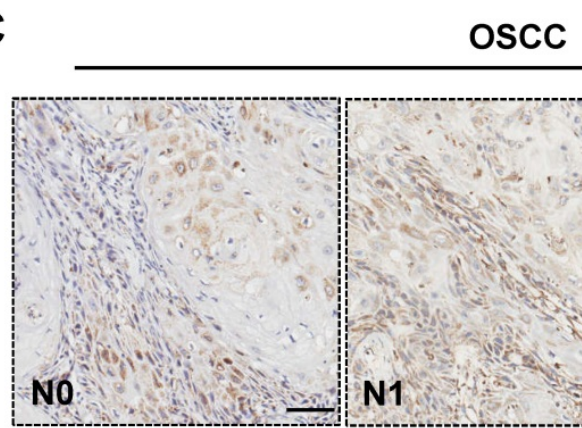

OSCC

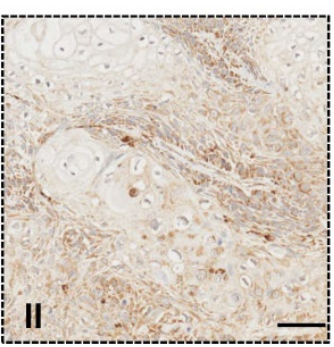

oscc

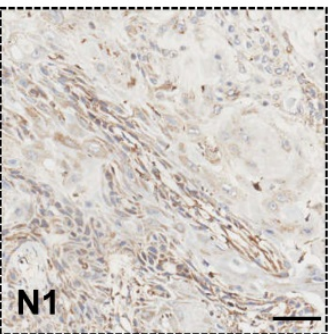

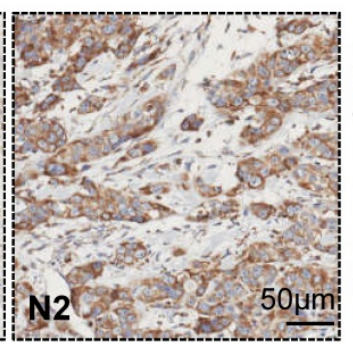

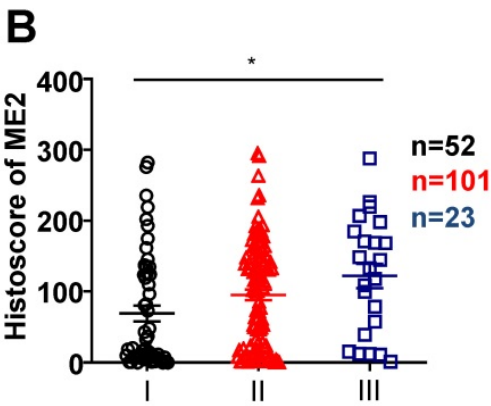

D

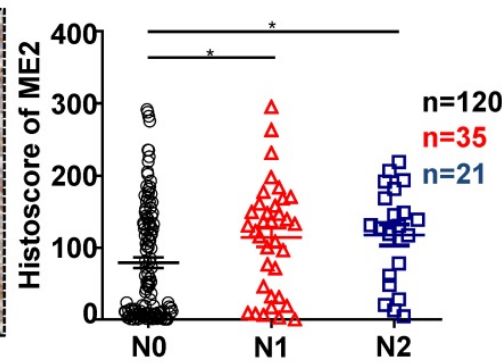

E

oscc
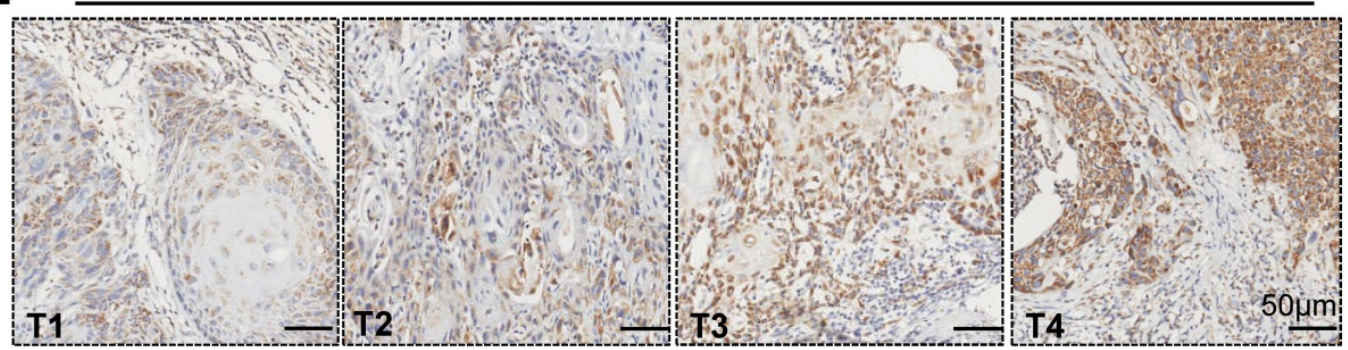

$\mathbf{F}$

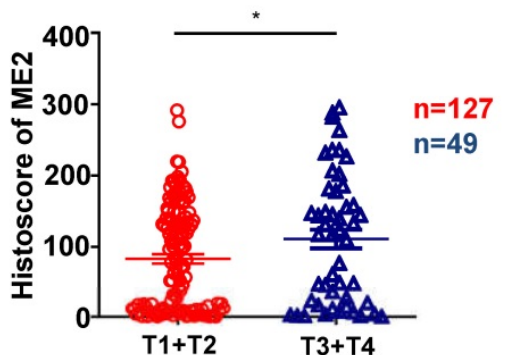

G

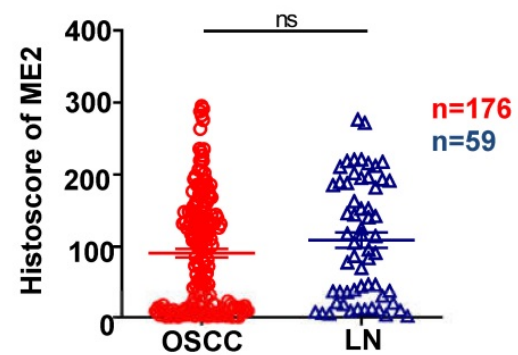

Figure 2. Human OSCC tissue array analysis demonstrated that ME2 is associated with high-grade, large-size and positive lymph node status OSCC tissue. Representative IHC images of ME2 in human oral cancer tissues with different grades (I-III, A), tumor size (T1-T4, E) and lymph node status (N0-N2, C). Quantitative analysis of the histoscore of ME2 expression in grade I, grade II and grade III tissues. These data suggested that ME2 expression levels in high-grade (grade III) OSCC tissues were more increased compared with low-grade (grade I) OSCC tissues (B). The expression of ME2 was related to larger tumor size (F). The expression of ME2 was related to positive lymphatic metastasis of human OSCC (D). There was no significant difference between the expression level of ME2 in primary OSCC and lymph node metastasis (G). 
A

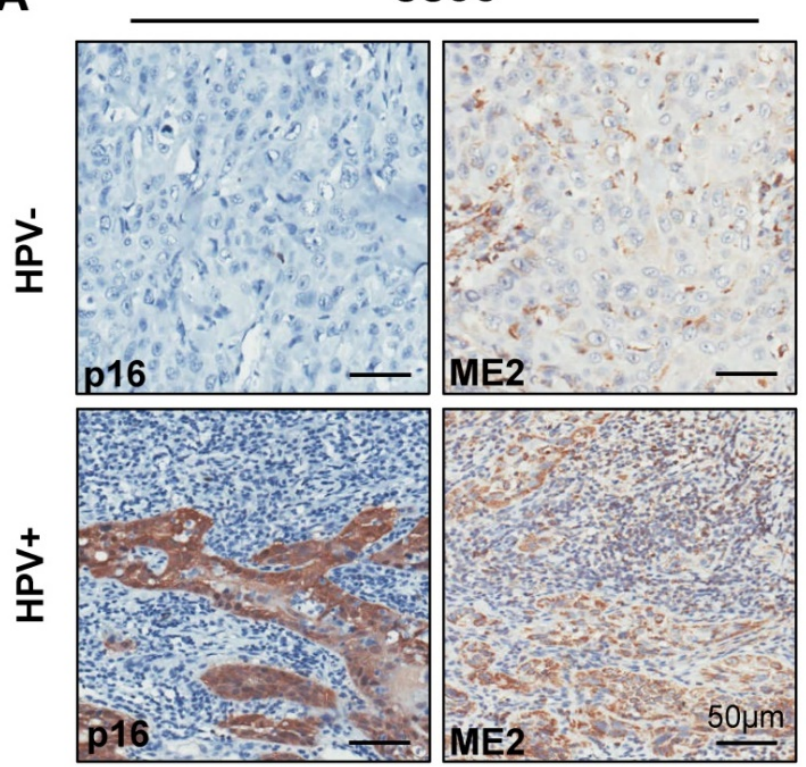

C

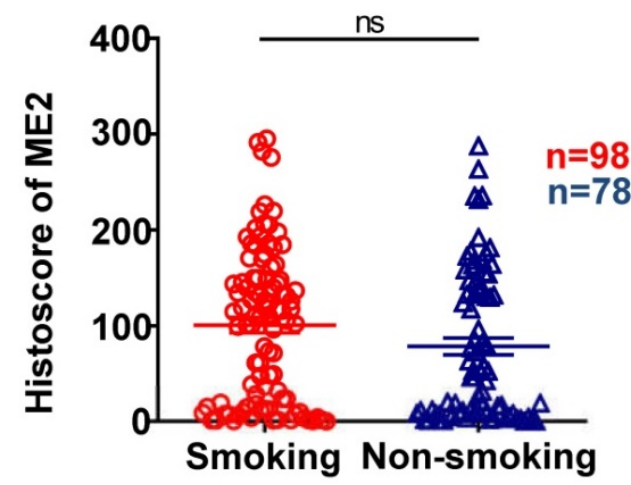

B

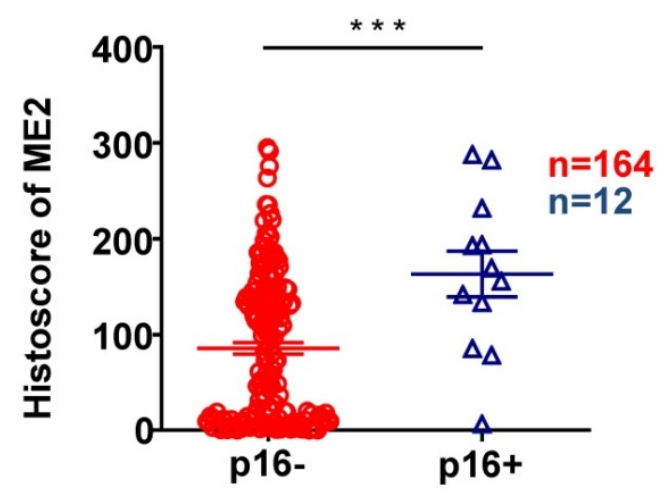

D

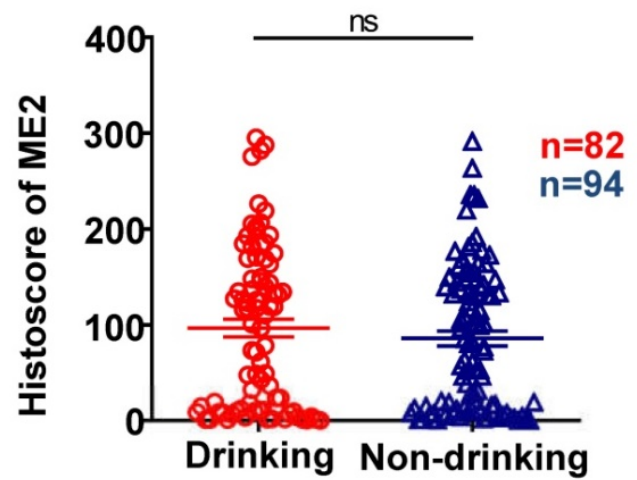

Figure 3. Representative IHC staining of 16 and IHC staining of ME2 in HPV positive human oral cancer tissues, and IHC staining of p16 and IHC staining of ME2 in HPV negative human oral cancer tissues (A). Quantitative analysis of ME2 in human oral cancer tissues between p16+ and p16- patients (B). Quantitative analysis of ME2 in human oral cancer tissues between smoking and nonsmoking patients $(C)$. Quantitative analysis of ME2 in human oral cancer tissues between drinking and nondrinking patients (D).

\section{The correlation between SOX2, Slug, ALDH1 and ME2 expression in human OSCC tissue}

Based on previously published studies, we carried out studies aiming to evaluate the correlation between the protein expression of ME2 and other proteins. ME2 may be correlated with Slug and other immune markers. We analyzed the quantification of immunohistochemical staining using Spearman's rank correlation coefficient. Fig. 4A shows the results obtained from the studies. Notable expression of SOX2, Slug, and ALDH1 was observed in human OSCC tissue. In addition, the immunohistochemical staining score suggested a close association between SOX2 (Fig. 4D), Slug (Fig. 4C), ALDH1 (Fig. 4B) and ME2 using hierarchical clustering analysis (Fig. 4E).

\section{Discussion}

Mounting evidence has indicated that ME2 plays a significant role in cancer cell[14-17, 27, 28]. In this study, we demonstrated that ME2 was highly upregulated in human primary OSCC tissue compared with normal mucosal tissue. The function and expression of ME2 in patients with malignancies, such as pancreatic cancer, Morris hepatomas and HNSCC, have been recently reported[15-17]. To the best of our knowledge, this was the first systematic medical research study of ME2 expression in human OSCC tissues. The most notable finding from this study was that overexpression of ME2 is correlated with high pathological grade, lymphatic metastasis, large tumor size and HPV. 

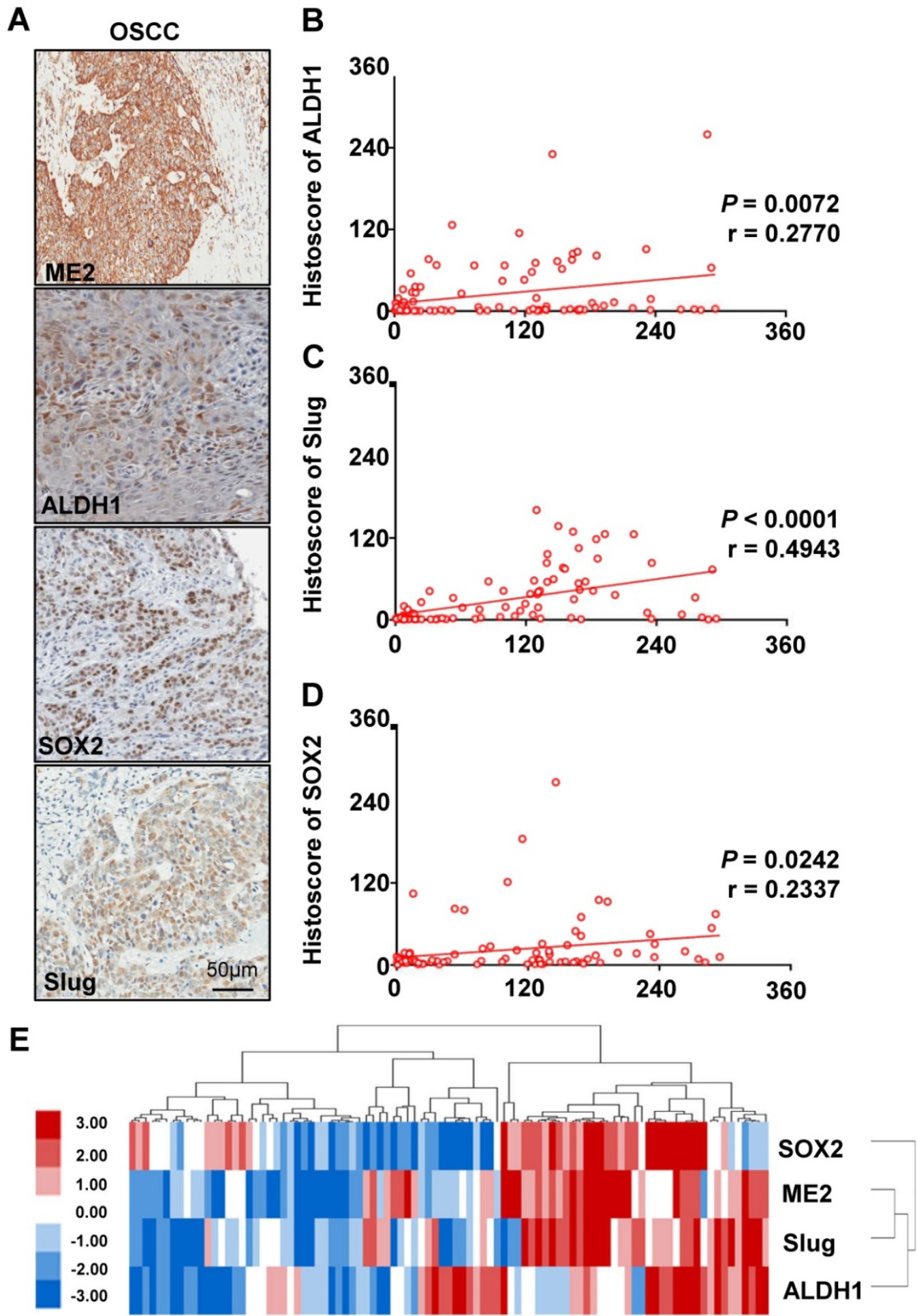

Figure 4. High expression levels of ALDH1, SOX2, Slug and ME2 in OSCC tissues. IHC of ME2, ALDH1, SOX2 and Slug in human OSCC tissues (A). Correlation of ME2 with ALDH1 (B), Slug (C), SOX2 (D) in human OSCC tissues. Hierarchical clustering of ME2, ALDH1, SOX2 and Slug immunohistochemical results in human OSCC tissues with statistics for mucosal, dysplasia and primary OSCC tissues (total $n=93)(E)$.

Many studies have shown that ME2 is detectable in many malignancies and predicts patient survival [15-17]. It was reported that ME2 is associated with poor survival in HNSCC [15]. However, only the effects of ME2 were investigated from the publicly available TCGA database. Therefore, we first compared the expression of ME2 with the survival rate in Chinese people's cohort using the best cutoff from Cutoff Finder, which suggests that the overexpression of ME2 may indicate a poor prognosis.

Previous studies on ME2 have suggested that the response to therapy mediated by ME2 expression has 
clinical significance [15]. However, the study of the expression of ME2 and its clinicopathological importance in OSCC is limited. The aim of this study was to investigate whether ME2 is overexpressed in human OSCC tissues. It is a fundamental connection to cancer pathogenesis between mitochondrial respiration and cancer cell dedifferentiation [29]. Therefore, overexpression of ME2 was significant, which indicated abnormal mitochondrial respiration may come about higher pathological grade and larger tumor size. In a previous study, ROS are upregulated in tumors and can lead to abnormal induction of signaling networks that cause tumorigenesis and metastasis [29]. Thus, positive lymph node status may express high level of ROS. It has been reported that depletion of ME2 inhibits ATP production, increases oxygen consumption and enhances ROS production [17]. ME2 is remarkably related to ROS-dependent signaling [17]. Hence, highly positive lymph node status was related to a high level of mitochondrial metabolism in human oral cancer tissues. Meanwhile positive lymph node status was significantly associated with the ME2 expression level, indicating that ME2 may play a role in the metabolism of OSCC.

Furthermore, a recent study reported that SOX2, Slug and ALDH are overexpressed in human head and neck cancer [30-33]. Our result indicated that a close association between SOX2, Slug, ALDH1 and ME2. In a previous study, AKT signaling is down regulated by knockdown of ME2 [17]. Afterwards, the AKT signaling pathway is the upstream signaling pathway of Slug [34]. The AKT signaling pathway can regulate EMT factors to activate the genes such as SOX2 and ALDH1 [35, 36]. Compared with our human OSCC tissue microarrays which may indicate that ME2 and Slug are negative costimulatory molecules that disrupt host antitumor immunity.

In conclusion, our results provide evidence that ME2 is overexpressed in OSCC tissue and is correlated with SOX2, Slug, and ALDH1, indicating that ME2 may be a potential target for OSCC. Nevertheless, the biomechanism of ME2 in OSCC was unclear. This problem remains to be solved.

\section{Supplementary Material}

Supplementary figure.

http://www.medsci.org/v17p0799s1.pdf

\section{Acknowledgements}

This work was supported by the National Natural Science Foundation of China (81874131, 81672668, and 81672667). Zhi-Jun Sun was supported by Hubei Province Natural Science Funds for Distinguished Young Scholar (2017CFA062).

\section{Competing Interests}

The authors have declared that no competing interest exists.

\section{References}

1. Siegel RL, Miller KD, Jemal A. Cancer statistics, 2018. CA: a cancer journal for clinicians. 2018; 68: 7-30.

2. Ali J, Sabiha B, Jan HU, Haider SA, Khan AA, Ali SS. Genetic etiology of oral cancer. Oral oncology. 2017; 70: 23-8.

3. Lee CE, Vincent-Chong VK, Ramanathan A, Kallarakkal TG, Karen-Ng LP, Ghani WM, et al. Collagen Triple Helix Repeat Containing-1 (CTHRC1) Expression in Oral Squamous Cell Carcinoma (OSCC): Prognostic Value and Clinico-Pathological Implications. Int J Med Sci. 2015; 12: 937-45.

4. Chi AC, Day TA, Neville BW. Oral cavity and oropharyngeal squamous cell carcinoma--an update. CA: a cancer journal for clinicians. 2015; 65: 401-21.

5. Fadlullah MZ, Chiang IK, Dionne KR, Yee PS, Gan CP, Sam KK, et al Genetically-defined novel oral squamous cell carcinoma cell lines for the development of molecular therapies. Oncotarget. 2016; 7: 27802-18.

6. Rhodus NL, Kerr AR, Patel K. Oral cancer: leukoplakia, premalignancy, and squamous cell carcinoma. Dent Clin North Am. 2014; 58: 315-40.

7. Galluzzi L, Kepp O, Vander Heiden MG, Kroemer G. Metabolic targets for cancer therapy. Nature reviews Drug discovery. 2013; 12: 829-46.

8. Teicher BA, Linehan WM, Helman LJ. Targeting cancer metabolism. Clinical cancer research : an official journal of the American Association for Cancer Research. 2012; 18: 5537-45.

9. Bobrovnikova-Marjon E, Hurov JB. Targeting metabolic changes in cancer: novel therapeutic approaches. Annu Rev Med. 2014; 65: 157-70.

10. Loeber G, Infante AA, Maurer-Fogy I, Krystek E, Dworkin MB. Human $\mathrm{NAD}(+)$-dependent mitochondrial malic enzyme. cDNA cloning, primary structure, and expression in Escherichia coli. J Biol Chem. 1991; 266: 3016-21.

11. Loeber G, Dworkin MB, Infante A, Ahorn H. Characterization of cytosolic malic enzyme in human tumor cells. FEBS Lett. 1994; 344: 181-6.

12. Loeber G, Maurer-Fogy I, Schwendenwein R. Purification, cDNA cloning and heterologous expression of the human mitochondrial NADP(+)-dependent malic enzyme. Biochem J. 1994; 304 ( Pt 3): 687-92.

13. Pongratz RL, Kibbey RG, Shulman GI, Cline GW. Cytosolic and mitochondrial malic enzyme isoforms differentially control insulin secretion. J Biol Chem. 2007; 282: 200-7

14. Jiang P, Du W, Mancuso A, Wellen KE, Yang X. Reciprocal regulation of p53 and malic enzymes modulates metabolism and senescence. Nature. 2013; 493: 689-93.

15. Woo SH, Yang LP, Chuang HC, Fitzgerald A, Lee HY, Pickering C, et al. Down-regulation of malic enzyme 1 and 2: Sensitizing head and neck squamous cell carcinoma cells to therapy-induced senescence. Head \& neck. 2016; 38 Suppl 1: E934-40.

16. Dey P, Baddour J, Muller F, Wu CC, Wang H, Liao WT, et al. Genomic deletion of malic enzyme 2 confers collateral lethality in pancreatic cancer. Nature. 2017; 542: 119-23.

17. Ren JG, Seth P, Clish CB, Lorkiewicz PK, Higashi RM, Lane AN, et al. Knockdown of malic enzyme 2 suppresses lung tumor growth, induces differentiation and impacts PI3K/AKT signaling. Scientific reports. 2014; 4: 5414.

18. Wu CC, Xiao Y, Li H, Mao L, Deng WW, Yu GT, et al. Overexpression of FAM3C is associated with poor prognosis in oral squamous cell carcinoma. Pathol Res Pract. 2019; 215: 772-78.

19. Ove R, Nabell LM. Induction chemotherapy for head and neck cancer: is there still a role? Future Oncol. 2016; 12: 1595-608

20. Sun ZJ, Chen G, Zhang W, Hu XA, Huang CF, Wang YF, et al. Mammalian Target of Rapamycin Pathway Promotes Tumor-Induced Angiogenesis in Adenoid Cystic Carcinoma: Its Suppression by Isoliquiritigenin through Dual Activation of c-Jun NH2-Terminal Kinase and Inhibition of Extracellular Signal-Regulated Kinase. J Pharmacol Exp Ther. 2010; 334: 500-12.

21. Huang CF, Zhang L, Ma SR, Zhao ZL, Wang WM, He KF, et al. Clinical significance of Keap1 and Nrf2 in oral squamous cell carcinoma. PloS one. 2013; 8: e83479.

22. He KF, Zhang L, Huang CF, Ma SR, Wang YF, Wang WM, et al. CD163+ tumor-associated macrophages correlated with poor prognosis and cancer stem cells in oral squamous cell carcinoma. Biomed Res Int. 2014; 2014: 838632.

23. Saldanha AJ. Java Treeview--extensible visualization of microarray data. Bioinformatics. 2004; 20: 3246-8.

24. Eisen MB, Spellman PT, Brown PO, Botstein D. Cluster analysis and display of genome-wide expression patterns. Proc Natl Acad Sci U S A. 1998; 95: 14863-8.

25. Sun ZJ, Zhang L, Hall B, Bian Y, Gutkind JS, Kulkarni AB. Chemopreventive and chemotherapeutic actions of mTOR inhibitor in genetically defined head and neck squamous cell carcinoma mouse model. Clinical cancer research : an official journal of the American Association for Cancer Research. 2012; 18: 5304-13.

26. Budczies J, Klauschen F, Sinn BV, Gyorffy B, Schmitt WD, Darb-Esfahani S, et al. Cutoff Finder: a comprehensive and straightforward Web application enabling rapid biomarker cutoff optimization. PloS one. 2012; 7: e51862. 
27. Ren JG, Seth P, Everett P, Clish CB, Sukhatme VP. Induction of erythroid differentiation in human erythroleukemia cells by depletion of malic enzyme 2. PloS one. 2010; 5.

28. Hsieh JY, Li SY, Tsai WC, Liu JH, Lin CL, Liu GY, et al. A small-molecule inhibitor suppresses the tumor-associated mitochondrial NAD(P)+-dependent malic enzyme (ME2) and induces cellular senescence. Oncotarget. 2015; 6: 20084-98.

29. Scatena R. Mitochondria and cancer: a growing role in apoptosis, cancer cell metabolism and dedifferentiation. Adv Exp Med Biol. 2012; 942: 287-308.

30. Fang J, Bao YY, Zhou SH, Luo XM, Yao HT, He JF, et al. Recurrent prognostic factors and expression of GLUT-1, PI3K and p-Akt in adenoid cystic carcinomas of the head and neck: Clinicopathological features and biomarkers of adenoid cystic carcinoma. Oncol Lett. 2012; 4: 1234-40.

31. Lundberg M, Renkonen S, Haglund C, Mattila PS, Leivo I, Hagstrom I, et al. Association of BMI-1 and p16 as prognostic factors for head and neck carcinomas. Acta Otolaryngol. 2016; 136: 501-5.

32. Seino S, Shigeishi H, Hashikata M, Higashikawa K, Tobiume K, Uetsuki R, et al. CD44(high) / ALDH1(high) head and neck squamous cell carcinoma cells exhibit mesenchymal characteristics and GSK3beta-dependent cancer stem cell properties. J Oral Pathol Med. 2016; 45: 180-8.

33. Baumeister P, Hollmann A, Kitz J, Afthonidou A, Simon F, Shakhtour I, et al. High Expression of EpCAM and Sox2 is a Positive Prognosticator of Clinical Outcome for Head and Neck Carcinoma. Scientific reports. 2018; 8: 14582.

34. Carpenter RL, Paw I, Dewhirst MW, Lo HW. Akt phosphorylates and activates HSF-1 independent of heat shock, leading to Slug overexpression and epithelial-mesenchymal transition (EMT) of HER2-overexpressing breast cancer cells. Oncogene. 2015; 34: 546-57.

35. Safa AR, Saadatzadeh MR, Cohen-Gadol AA, Pollok KE, Bijangi-Vishehsaraei K. Emerging targets for glioblastoma stem cell therapy. J Biomed Res. 2016; 30: 19-31.

36. Sun C, Sun L, Li Y, Kang X, Zhang S, Liu Y. Sox2 expression predicts poor survival of hepatocellular carcinoma patients and it promotes liver cancer cell invasion by activating Slug. Med Oncol. 2013; 30: 503 